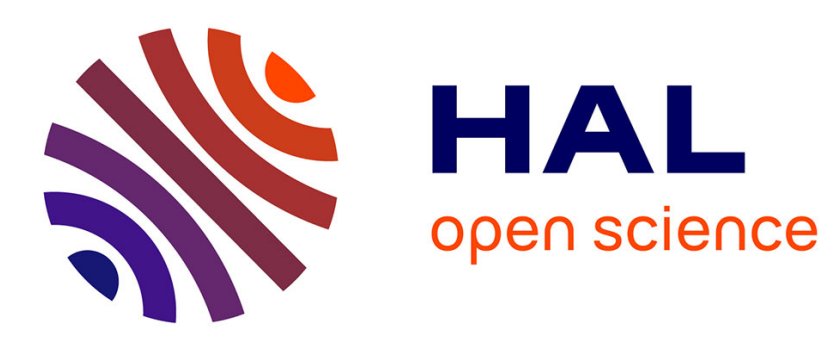

\title{
On the shell theory on the nanoscale with surface stresses
}

Holm Altenbach, Victor A. Eremeyev

\section{To cite this version:}

Holm Altenbach, Victor A. Eremeyev. On the shell theory on the nanoscale with surface stresses. International Journal of Engineering Science, 2011, 49 (12), pp.1294-1301. hal-00824104

\section{HAL Id: hal-00824104 \\ https://hal.science/hal-00824104}

Submitted on 21 May 2013

HAL is a multi-disciplinary open access archive for the deposit and dissemination of scientific research documents, whether they are published or not. The documents may come from teaching and research institutions in France or abroad, or from public or private research centers.
L'archive ouverte pluridisciplinaire HAL, est destinée au dépôt et à la diffusion de documents scientifiques de niveau recherche, publiés ou non, émanant des établissements d'enseignement et de recherche français ou étrangers, des laboratoires publics ou privés. 


\title{
On the shell theory on the nanoscale with surface stresses
}

\author{
Holm Altenbach ${ }^{\mathrm{a}, 1}$, Victor A. Eremeyev ${ }^{\mathrm{a}, \mathrm{b}, *}$
}

${ }^{a}$ Martin Luther University, Halle Wittenberg, Halle (Saale), Germany
${ }^{\mathrm{b}}$ South Scientific Center of RASci \& South Federal University, Rostov on Don, Russia

In Memory of Professor A. Cemal Eringen.

\section{Keywords:}

Surface stresses

Cosserat shell

Nonlinear elasticity

Micropolar elasticity

6 Parametric shell theory

Nanosized shells and plates

\section{A B S T R A C T}

Below we discuss the derivation of the governing nonlinear shell equations taking into account the surface stresses. The surface effects are significant for the modeling of some structures as nanofilms, nanoporous materials and other nano size structures. In particu lar, the surface stresses are responsible for the size effect, i.e. dependence of the material properties on the specimen size. The theory of elasticity with surface stresses is applied to the modeling of shells with nano scaled thickness. It will be shown that the resultant stress and couple stress tensors can be represented as a sum of two terms. The first term in the sum depends on the stress distribution in the bulk material while the second one relates to the surface stresses. Hence, the resultant stress and couple stress tensors are lin ear functions with respect to the surface stresses. As an example the effective stiffness properties of a linear elastic Cosserat shells taking into account the surface stresses are presented.

\section{Introduction}

The development of nanotechnologies extends the field of application of the classical or non-classical theories of plates and shells towards the new thin-walled structures, such as nanofilms, nanotubes, fullerenes, etc. In general, modern nanomaterials have physical properties which can be different from the properties of the bulk materials. This can be explained by the importance of the surface effects which is much higher in comparison with classical applications. The classical elasticity can be extended to the nanoscale by implementation of the theory of elasticity taking into account the surface stresses, cf. Duan, Wang, and Karihaloo (2008) among others. In particular, the surface stresses are responsible for the size-effect, that means the material properties of a specimen depend on its size. For example, the Young's modulus of a cylindrical specimen increases significantly, when the cylinder diameter becomes very small (Chen, Shi, Zhang, Zhu, \& Yan, 2006; Cuenot, Frétigny, Demoustier-Champagne, \& Nysten, 2004; Guo \& Zhao, 2007; Jing et al., 2006; Yun \& Park, 2009; Zheng, Cao, Li, Feng, \& Wang, 2010). The surface stresses are the generalization of the scalar surface tension which is a well-known phenomenon in the theory of capillarity, (see e.g. Landau \& Lifshitz, 1987). The investigations of the surface phenomena were initiated by Gibbs (1928), Laplace $(1805,1806)$ and Young $(1805)$. The current state of the art is presented in some reviews (see e.g. Duan et al., 2008; Finn, 1986; Müller \& Saúl, 2004; Orowan, 1970; Podstrigach \& Povstenko, 1985; Rusanov, 2005a, 2005b). The nonlinear continuum mechanics of elastic solids with surface stresses was developed by Gurtin and Murdoch (1975a, 1975b) and extended by Steigmann and Ogden (1999) with regard of the surface bending stiffness.

* Corresponding author at: South Scientific Center of RASci \& South Federal University, Rostov on Don, Russia. Tel.: +7 8632996628.

E mail addresses: holm.altenbach@iw.uni halle.de (H. Altenbach), eremeyev.victor@gmail.com (V.A. Eremeyev).

${ }^{1}$ Tel.: +49 (0) 345 5528430/1; fax: +49 (0) 3455527359 . 
For such nano-objects as nanofilms, nanoshells, nanowires, etc., it is quite natural to use the one-dimensional (1D) and two-dimensional (2D) models of the mechanics of structures, i.e. the theories of rods, beams, plates, and shells. Let us note that there exist various approaches to formulate these theories especially in the case of shells and plates. The discussion on the derivation of the 1D and 2D equations is very extensive (see Altenbach \& Zhilin, 1988; Chróścielewski, Makowski, \& Pietraszkiewicz, 2004; Eremeyev \& Zubov, 2008; Grigolyuk \& Kogan, 1972; Grigolyuk \& Selezov, 1973; Libai \& Simmonds, 1998; Naghdi, 1972; Rubin, 2000; Wunderlich, 1973) among others. The approaches can be classified, for example, by the starting point of the derivation. This can be the well-known three-dimensional continuum mechanics equations together with the application of engineering hypotheses or mathematical techniques. In contrast, one can introduce à priory a deformable surface (two-dimensional manifold) which is the basis for a more natural formulation of the two-dimensional governing equations. This so-called direct approach should be supplemented by the theoretical or experimental identification of the material parameters in the constitutive equations. The known plate and shell models are related to the names of Kirchhoff, Love, Cosserat, von Kàrmàn, Timoshenko, Vlasov, Reissner, Mindlin, Koiter, Naghdi, and Reddy among others. For example, in the literature are known various models of shells related to the Cosserat model, see the recent review (Altenbach, Altenbach, \& Eremeyev, 2010). In particular, using the through-the-thickness integration of the equilibrium equations of micropolar plate-like body, Eringen proposed the linear theory of micropolar plates (Eringen, 1967). Most of these theories are used in modeling the nanosized structures, see, for example, (Altenbach, Eremeyev, \& Morozov, 2009; Dahmen, Lehwald, \& Ibach, 2000; Eremeyev, Altenbach, \& Morozov, 2009; Guo \& Zhao, 2005; He, Lim, \& Wu, 2004; Huang, 2008; Kudin, Scuseria, \& Yakobson, 2001; Lu, He, Lee, \& Lu, 2006, 2009; Miller \& Shenoy, 2000; Peng, Wu, Hwang, Song, \& Huang, 2008; Shi, Guo, \& Ru, 2008; Wang, Huang, \& Yu, 2008; Wang, Zhao, \& Huang, 2010; Zhu, 2010), where various 1D and $2 \mathrm{D}$ theories are used. In particular, the theory of elasticity with surface stresses is applied to the modifications of the twodimensional theories of nanosized plates and shells, see (Altenbach et al., 2009; Dahmen et al., 2000; Eremeyev et al., 2009; Huang, 2008; Lu et al., 2006; Wang \& Zhao, 2009; Zhu, Wang, \& Karihaloo, 2009).

In this paper we use the general nonlinear theory of shells presented in Libai and Simmonds (1998), Chróścielewski et al. (2004), Eremeyev and Zubov (2008) for the modification of the constitutive equations of a shell taking into account the surface stresses. In some references this variant of the theory of shells is also named the micropolar shell theory (Altenbach et al., 2010) because it is kinematically equivalent to the 2D Cosserat (micropolar) continuum, i.e. 2D medium with independent translations and rotations which origins are stresses and couple stresses, respectively. The considered shell theory was initiated by the Cosserat brothers within framework of the direct approach, see the fundamental centenarian book (Cosserat \& Cosserat, 1909). The foundation of 3D theory of the micropolar continuum is given by Kafadar and Eringen (1971), Kafadar and Eringen (1976), and Eringen $(1966,1999)$ among others.

The paper is organized as follows. In Section 2 we recall the basic equations of the micropolar shells theory. Then in Section 3 we formulate the equations of equilibrium for a nonlinear elastic shell-like body with surface stresses. In Section 4 the reduction procedure from the $3 \mathrm{D}$ equilibrium equations to the $2 \mathrm{D}$ ones is discussed. Here we obtain the modified constitutive equations for the stress and couple stress resultants tensors taking into account the surface stresses. We show that both the stress and the couple stress resultant tensors may be represented as a sum of two terms. The first term is the volume stress resultant while the second one is determined by the surface stresses and the shell geometry. Finally, in Section 5 we consider the modification of the constitutive relations in the case of small strains. In the linear case this modification reduces to the addition of new terms to the elastic stiffness parameters. The influence of these terms on the bending stiffness of a shell is discussed. We show that the surface elasticity makes the shell more stiffer in comparison with the shell without surface stresses. In the derivations the direct tensor notation is applied as suggested in the books (Lebedev, Cloud, \& Eremeyev, 2010; Lurie, 2005).

\section{Basic equations of the 6-parametric elastic shell theory}

Following (Altenbach et al., 2010; Eremeyev, 2005; Eremeyev \& Zubov, 2008) we recall the basic equations of the nonlinear micropolar shell theory. We consider a micropolar shell as a deformable surface, each point of which has 6 degrees of freedom, i.e. three translational degrees and three rotational ones. The interaction between different parts of the shell is described by forces and moments only. With other words, a micropolar shell is kinematically equivalent to a twodimensional Cosserat or micropolar continuum, which can be introduced on the base of the 3D theory presented in Kafadar and Eringen (1971), Kafadar and Eringen (1976), Eringen (1999). The kinematical model of the shell is based on the introduction of a directed material surface $\omega$, which is determined in the actual configuration by

$$
\mathbf{r}\left(q^{1}, q^{2}\right), \quad \mathbf{d}_{k}\left(q^{1}, q^{2}\right) ; \quad \mathbf{d}_{k} \cdot \mathbf{d}_{m}=\delta_{k m},
$$

where $\mathbf{r}\left(q^{1}, q^{2}, t\right)$ is the position vector defining the geometry of $\omega, q^{1}, q^{2} \in \omega$ are coordinates on $\omega, \mathbf{d}_{k}\left(q^{1}, q^{2}\right)$ are orthonormal vectors called directors, $k=1,2,3$, and $\delta_{k m}$ is the Kronecker's symbol.

In the reference configuration the shell is described by the surface $\Omega$ with the position vector and directors

$$
\mathbf{R}\left(q^{1}, q^{2}\right), \quad \mathbf{D}_{k}\left(q^{1}, q^{2}\right) ; \quad \mathbf{D}_{k} \cdot \mathbf{D}_{m}=\delta_{k m} \quad(k=1,2,3) .
$$

Introducing the proper orthogonal tensor $\mathbf{Q}$ by the relation

$$
\mathbf{Q}=\mathbf{D}_{k} \otimes \mathbf{d}_{k},
$$


which is also named the microrotation tensor (Eringen, 1999), we describe the deformation of a micropolar shell by two fields

$$
\boldsymbol{r}=\boldsymbol{r}\left(q^{1}, q^{2}\right) \equiv \boldsymbol{R}\left(q^{1}, q^{2}\right)+\mathbf{v}\left(q^{1}, q^{2}\right), \quad \mathbf{Q}=\mathbf{Q}\left(q^{1}, q^{2}\right),
$$

where $\mathbf{v}$ is the displacement vector.

For an elastic shell there exists the surface strain energy density $\mathcal{W}$ which is given by the following formula

$$
\mathcal{W}=\mathcal{W}\left(\mathbf{F}, \mathbf{Q}, \nabla_{s} \mathbf{Q}\right)
$$

where

$$
\begin{aligned}
& \mathbf{F}=\nabla_{S} \mathbf{r}, \quad \nabla_{S}(\cdots)=\mathbf{R}^{\alpha} \otimes \frac{\partial(\cdots)}{\partial q^{\alpha}}, \quad \alpha, \beta=1,2, \\
& \mathbf{R}^{\alpha} \cdot \mathbf{R}_{\beta}=\delta_{\beta}^{\alpha}, \quad \mathbf{R}^{\alpha} \cdot \mathbf{N}=0, \quad \mathbf{R}_{\alpha}=\frac{\partial \mathbf{R}}{\partial q^{\alpha}} .
\end{aligned}
$$

In Eqs. (2) F is the surface deformation gradient, $\nabla_{S}$ is the surface nabla-operator in the reference configuration, $\mathbf{R}_{\alpha}, \mathbf{R}^{\beta}$ are the primary and reciprocal basis on the shell surface $\Omega$ in the reference configuration, and $\mathbf{N}$ is the unit normal to $\Omega$.

After application of the principle of the frame indifference (Truesdell \& Noll, 1965) the function $\mathcal{W}$ takes the form

$$
\begin{aligned}
& \mathcal{W}=\mathcal{W}(\mathbf{E}, \mathbf{K}), \\
& \mathbf{E}=\mathbf{F} \cdot \mathbf{Q}^{T}, \quad \mathbf{K}=\frac{1}{2} \mathbf{R}^{\alpha} \otimes\left(\frac{\partial \mathbf{Q}}{\partial q^{\alpha}} \cdot \mathbf{Q}^{T}\right)_{\times} .
\end{aligned}
$$

Here $\mathbf{E}$ and $\mathbf{K}$ are the Lagrangian strain measures of a shell, which can be introduced by various approaches by analogy with the 3D Cosserat Continuum (see Pietraszkiewicz \& Eremeyev, 2009), and $(\cdots)_{\times}$denotes the vectorial invariant of the secondorder tensor (Lebedev et al., 2010).

The Lagrangian equilibrium equations are given by

$$
\begin{aligned}
& \nabla_{S} \cdot \mathbf{D}+\mathbf{q}=\mathbf{0}, \quad \nabla_{S} \cdot \mathbf{H}+\left[\mathbf{F}^{T} \cdot \mathbf{D}\right]_{\times}+\mathbf{c}=\mathbf{0}, \\
& \mathbf{D}_{S}=\frac{\partial \mathcal{W}}{\partial \mathbf{E}} \cdot \mathbf{Q}, \quad \mathbf{H}=\frac{\partial \mathcal{W}}{\partial \mathbf{K}} \cdot \mathbf{Q} .
\end{aligned}
$$

$\mathbf{D}$ and $\mathbf{H}$ are the surface stress and stress couple resultant tensors of the first Piola-Kirchhoff type, while $\mathbf{q}$ and $\mathbf{c}$ are the external surface force and moment vectors, respectively. The strain measures $\mathbf{E}$ and $\mathbf{K}$ are work-conjugate to the respective stress measures $\mathbf{D}$ and $\mathbf{H}$.

Let us assume the isotropic material behavior. In this case the surface strain energy density can be written as follows (Chróścielewski et al., 2004; Eremeyev \& Pietraszkiewicz, 2006; Eremeyev \& Zubov, 2008)

$$
2 W=\alpha_{1} \operatorname{tr}^{2} \mathbf{E}_{\|}+\alpha_{3} \operatorname{tr}\left(\mathbf{E}_{\|} \cdot \mathbf{E}_{\|}^{T}\right)+\alpha_{4} \mathbf{N} \cdot \mathbf{E}^{T} \cdot \mathbf{E} \cdot \mathbf{N}+\beta_{1} \operatorname{tr}^{2} \mathbf{K}_{\|}+\beta_{3} \operatorname{tr}\left(\mathbf{K}_{\|} \cdot \mathbf{K}_{\|}^{T}\right)+\beta_{4} \mathbf{N} \cdot \mathbf{K}^{T} \cdot \mathbf{K} \cdot \mathbf{N} .
$$

Here $\mathbf{E}_{\|}=\mathbf{E} \cdot \mathbf{A}, \mathbf{K}_{\|}=\mathbf{K} \cdot \mathbf{A}, \mathbf{A}=\mathbf{I} \quad \mathbf{N} \otimes \mathbf{N}$ is the surface unit tensor, I is the unit tensor, and $\alpha_{i}, \beta_{i}$ are the elastic parameters given by

$$
\begin{aligned}
& \alpha_{1}=C v, \quad \alpha_{2}=0, \quad \alpha_{3}=C(1 \quad v), \quad \alpha_{4}=\alpha_{s} C(1 \quad v), \\
& \beta_{1}=D v, \quad \beta_{2}=0, \quad \beta_{3}=D(1 \quad v), \quad \beta_{4}=\alpha_{t} D(1 \quad v), \\
& C=\frac{E h}{1-v^{2}}, \quad D=\frac{E h^{3}}{12\left(1-v^{2}\right)},
\end{aligned}
$$

where $E$ and $v$ are the Young's modulus and the Poisson's ratio of the bulk material, respectively, $\alpha_{s}$ and $\alpha_{t}$ are dimensionless coefficients, while $h$ is the shell thickness. The number $\alpha_{s}$ is similar to the shear correction factor introduced by Reissner (1944), Reissner (1945) $\left(\alpha_{s}=5 / 6\right)$ and Mindlin (1951) $\left(\alpha_{s}=\pi^{2} / 12\right)$. The value $\alpha_{t}=0.7$ was proposed by Pietraszkiewicz (1979a, 1979b).

In the case of infinitesimal strains the theory of micropolar shells is presented in Chróścielewski et al. (2004), Eremeyev and Zubov (2008), Lebedev et al. (2010). As in the 3D micropolar continuum instead of the microrotation tensor $\mathbf{Q}$ we introduce the microrotation vector $\boldsymbol{\theta}$ and use the displacement vector $\mathbf{v}$, so we have

$$
\mathbf{F} \approx \mathbf{A}+\nabla_{S} \mathbf{v}, \quad \mathbf{Q} \approx \mathbf{I}+\mathbf{I} \times \boldsymbol{\theta}
$$

and the strain measures become

$$
\mathbf{E}=\nabla_{S} \mathbf{v}+\mathbf{A} \times \boldsymbol{\theta}, \quad \mathbf{K}=\nabla_{S} \boldsymbol{\theta} .
$$

Eqs. (7) are the analogues of the strain measures in the linear micropolar elasticity (Eringen, 1999).

\section{Equilibrium of an elastic body with surface stresses}

The finite deformations of the elastic solid with surface stresses are discussed in Gurtin and Murdoch (1975a). Following (Gurtin \& Murdoch, 1975a) let us consider the boundary-value problem for a shell-like body, see Fig. 1. The equilibrium equations and the boundary conditions of the elastic body with surface stresses are given by

$$
\nabla_{\mathbf{x}} \cdot \mathbf{P}+\rho \mathbf{f}=\mathbf{0},\left.\quad\left(\mathbf{N}_{ \pm} \cdot \mathbf{P} \quad \nabla_{S}^{ \pm} \cdot \mathbf{S}_{ \pm}\right)\right|_{\Omega_{ \pm}}=\mathbf{t}_{ \pm},\left.\quad \mathbf{u}\right|_{\Omega_{u}}=\mathbf{0},\left.\quad \mathbf{N} \cdot \mathbf{P}\right|_{\Omega_{f}}=\mathbf{t} .
$$




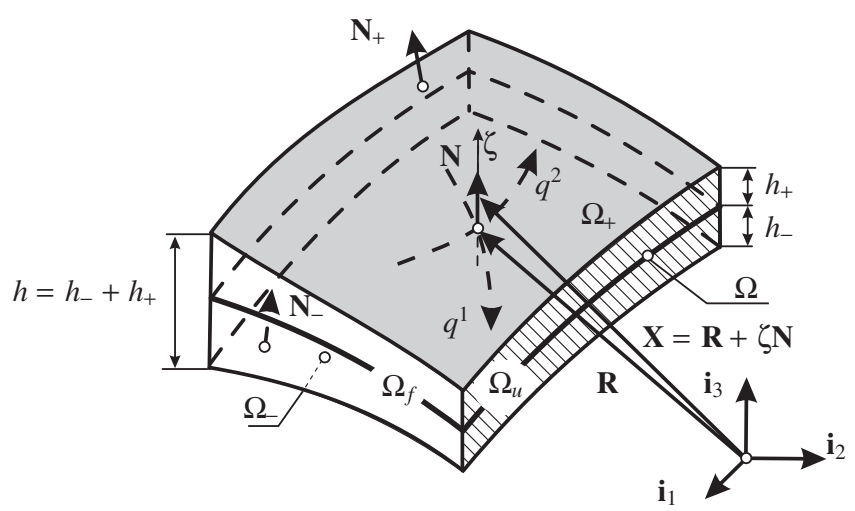

Fig. 1. Shell like body in the reference configuration.

Here $\mathbf{P}$ is the first Piola-Kirchhoff stress tensor, $\nabla_{\mathbf{x}}$ is the 3D nabla operator in the reference configuration, $\mathbf{S}_{ \pm}$are the surface stress tensors of the first Piola-Kirchhoff type acting on the surfaces $\Omega_{ \pm}, \mathbf{u}=\mathbf{X} \quad \mathbf{X}$ is the displacement vector, $\mathbf{x}$ and $\mathbf{X}$ are the position vectors in the actual and reference configurations, respectively, $\mathbf{f}$ and $\mathbf{t}_{ \pm}, \mathbf{t}$ are the body force and surface load vectors, respectively, and $\rho$ is the density in the reference configuration. We assume that the part of the body lateral surface $\Omega_{u}$ is fixed, while on $\Omega_{f}$ the surface stresses are absent. The corresponding actual configuration of the shell-like body is given in Fig. 2.

Introducing the base surface of the shell-like body $\Omega$ we use the following standard representation of the position vector $\mathbf{X}$ (see e.g. Lebedev et al., 2010):

$$
\mathbf{X}=\mathbf{X}\left(q^{1}, q^{2}, \zeta\right) \equiv \mathbf{r}\left(q^{1}, q^{2}\right)+\zeta \mathbf{N}\left(q^{1}, q^{2}\right),
$$

where $q^{1}$ and $q^{2}$ are coordinates on $\Omega$, and $\zeta$ is the normal coordinate, $\zeta \in\left[\quad h_{-}, h_{+}\right], h=h_{-}+h_{+}$is the thickness. Hence, the set $\left\{q^{1}, q^{2}, \zeta\right\}$ constitutes so-called shell coordinates which are the Lagrangian coordinates in 3D space, and $\mathbf{x}=\mathbf{x}\left(q^{1}, q^{2}, \zeta\right)$. Within these coordinates the spatial nabla-operator is given by Lebedev et al. (2010)

$$
\nabla_{\mathbf{x}}=\mathbf{X}^{\alpha} \frac{\partial}{\partial q^{\alpha}}+\mathbf{N} \frac{\partial}{\partial \zeta}=\left(\begin{array}{ll}
\mathbf{A} & \zeta \mathbf{B}
\end{array}\right)^{-1} \cdot \mathbf{R}^{\alpha} \frac{\partial}{\partial q^{\alpha}}+\mathbf{N} \frac{\partial}{\partial z}=\left(\begin{array}{ll}
\mathbf{A} & z \mathbf{B}
\end{array}\right)^{-1} \cdot \nabla_{S}+\mathbf{N} \frac{\partial}{\partial \zeta} .
$$

Here $\mathbf{B}$ is the curvature tensor of $\Omega, \mathbf{B}=\nabla_{S} \mathbf{N}$, and $\mathbf{X}^{\alpha}, \mathbf{N}$ is the reciprocal basis. Note that the surface nabla-operators $\nabla_{S}^{ \pm}$ depend on the surfaces $\Omega_{ \pm}$, and is determined by the formulae

$$
\nabla_{S}^{ \pm}=\left(\mathbf{A} \mp h_{ \pm} \mathbf{B}\right)^{-1} \cdot \nabla_{S} .
$$

For $\mathbf{P}$ we use the standard constitutive equation of the nonlinear elasticity

$$
\mathbf{P}=\frac{\partial \mathcal{U}}{\partial \nabla_{\mathbf{x}} \mathbf{x}},
$$

where $\mathcal{U}=\mathcal{U}\left(\nabla_{\mathbf{x}} \mathbf{x}\right)$ is the strain energy density function. In the theory presented in Gurtin and Murdoch (1975a) the tensors $\mathbf{S}_{ \pm}$are similar to the membrane stress resultants and defined by

$$
\mathbf{S}_{ \pm}=\frac{\partial \mathcal{U}_{ \pm}}{\partial \mathbf{F}_{ \pm}}
$$

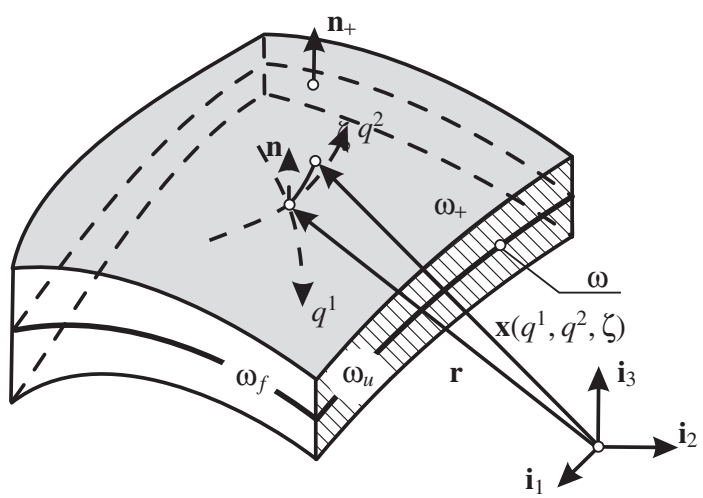

Fig. 2. Shell like body in the actual configuration. 
where $\mathcal{U}_{ \pm}$are the surface strain energy densities, and $\mathbf{F}_{ \pm}=\nabla_{S}^{ \pm} \mathbf{x}_{ \pm}$are the surface deformation gradients estimated on $\Omega_{ \pm}$, $\mathbf{x}_{ \pm}=\left.\mathbf{x}\right|_{\zeta= \pm h / 2}$ the position vectors of $\Omega_{ \pm}$.

Remark 1. In the theory of elasticity with surface stresses it is assumed the compatibility conditions (see Gurtin \& Murdoch, 1975a; Podstrigach \& Povstenko, 1985; Steigmann \& Ogden, 1999)

$$
\mathbf{x}_{ \pm}=\left.\mathbf{X}\right|_{\zeta \pm h / 2}
$$

i.e. the surface displacement are equal the limit of the displacements of the bulk material. In other words we assume that the surface displacements describe the deformation of a surface layer of infinitesimal thickness. If we consider the surface stresses as a model of stresses acting in the surface layer of small thickness then the mean displacement of this layer does not coincide with the limit of 3D displacement vector. Hence, the latter equation seems to be satisfactory in the case of smooth surfaces while for rough or fractal-like surfaces it should be replaced by more general equation

$$
\mathbf{x}_{ \pm}=\mathcal{A}\left(\left.\mathbf{x}\right|_{\zeta \pm h / 2}\right),
$$

where $\mathcal{A}$ is a nonlinear operator, in general. The properties of $\mathcal{A}$ depend on the surface structure.

\section{Reduction from 3D to 2D}

There are many possibilities to reduce the 3D equilibrium equations to the two-dimensional one. In particular, the procedure in Libai and Simmonds (1998), Chróścielewski et al. (2004) reduces the non-linear 3D equations of motion to the exact $2 \mathrm{D}$ equations. For the nonlinear elastic body without surface stresses, i.e. when $\mathbf{S}_{ \pm}=\mathbf{0}$, this technique gives the following relations between $\mathbf{D}, \mathbf{H}$, and $\mathbf{P}$ :

$$
\mathbf{D}=\int \mathbf{G} \cdot \mathbf{P} d \zeta, \quad \mathbf{H}=\int \mathbf{G} \cdot \mathbf{P} \times \mathbf{z} d \zeta, \quad \int(\cdots) d \zeta=\int_{h}^{h_{+}}(\cdots) d \zeta
$$

where $\mathbf{z}$ is the base reference deviation and $\mathbf{G} \equiv \mathbf{N} \times\left(\begin{array}{ll}\mathbf{A} & \zeta \mathbf{B}\end{array}\right) \times \mathbf{N}$ is the geometrical tensor defined in Libai and Simmonds (1998). The surface loads $\mathbf{q}$ and $\mathbf{c}$ in (4) are also determined by the through-the-thickness integration procedure.

Note that the boundary value problem (8) is linear with respect to the surface stresses $\mathbf{S}_{ \pm}$, and the through-the-thickness integration procedure is linear too. This means that the stress resultants for the shell with surface stresses can be represented as a sum of two terms

$$
\mathbf{D}^{*}=\mathbf{D}+\mathbf{D}_{S} \quad \mathbf{H}^{*}=\mathbf{H}+\mathbf{M}_{s},
$$

where $\mathbf{D}$ and $\mathbf{H}$ are the classical stress and couple stress resultant tensors given by (9), while $\mathbf{D}_{S}$ and $\mathbf{H}_{S}$ are the resultant tensors induced by $\mathbf{S}_{ \pm}$

$$
\mathbf{D}_{S}=G_{+} \nabla_{S} \cdot \mathbf{S}_{+}+G_{-} \nabla_{S} \cdot \mathbf{S}_{-} \quad \mathbf{M}_{S}=\frac{h}{2}\left[G_{+}\left(\nabla_{S} \cdot \mathbf{S}_{+}\right) \times \mathbf{z}_{+} \quad G_{-}\left(\nabla_{S} \cdot \mathbf{S}_{-}\right) \times \mathbf{z}_{-}\right] .
$$

Here $G \equiv \operatorname{det}(\mathbf{A} \quad \zeta \mathbf{B})=1 \quad 2 M \zeta+K \zeta^{2}$ is the geometric scale factor (Libai \& Simmonds, 1998; Naghdi, 1972), $M$ and $K$ are the mean and Gaussian curvatures of $\Omega$, and $\mathbf{z}_{ \pm}=\left.\mathbf{z}\right|_{\zeta= \pm h / 2}, G_{ \pm}=\left.G\right|_{\zeta= \pm h / 2}$. The representation (10) means that the reduction

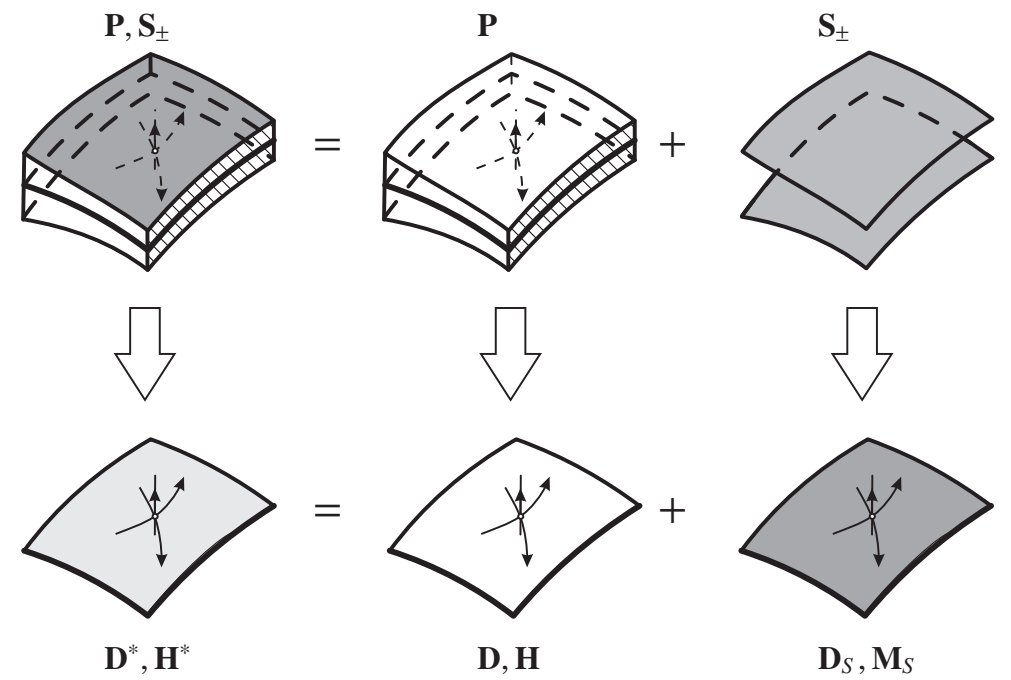

Fig. 3. Scheme of the reduction procedure, $\mathbf{D}^{*} \quad \mathbf{D}+\mathbf{D}_{S}$ and $\mathbf{H}^{*} \quad \mathbf{H}+\mathbf{M}_{S}$. 
procedure for the shell-like body can be applied for body made of the bulk material and for two surfaces independently. This is illustrated in Fig. 3. In particular, one can apply any classical reduction procedure used in the theory of shells, and then add new terms in the stress measures which depend on surface stresses.

To illustrate this representation and to avoid awkward calculations, further we restrict ourselves assuming both the linear theory and the plane geometry $(\mathbf{B}=\mathbf{0})$.

\section{Linear theory of micropolar plates with surface stresses}

For the infinitesimal strains the surface stress tensors $\mathbf{S}_{ \pm}$are given by Gurtin and Murdoch (1975a), Duan et al. (2008)

$$
\mathbf{S}_{ \pm}=\lambda_{S}^{ \pm} \mathbf{A t r} \mathbf{e}_{ \pm}+2 \mu_{S}^{ \pm} \mathbf{e}_{ \pm}, \quad 2 \mathbf{e}_{ \pm}=\nabla_{S} \mathbf{u}_{ \pm} \cdot \mathbf{A}+\mathbf{A} \cdot\left(\nabla_{S} \mathbf{u}_{ \pm}\right)^{T},
$$

where $\mathbf{u}_{ \pm}=\left.\mathbf{u}\right|_{\zeta \pm h / 2}, \lambda_{S}^{ \pm}$and $\mu_{S}^{ \pm}$are the surface analogues of the Lamé's constants. Further for the sake of simplicity we consider the symmetric case when $\lambda_{s}^{ \pm}=\lambda_{s}$ and $\mu_{s}^{ \pm}=\mu_{s}$.

Taking into account (12) and following (Altenbach et al., 2009; Eremeyev et al., 2009) we obtain the relations for the effective stiffness properties

$$
\begin{aligned}
& \alpha_{1}=C v+2 \lambda_{S}, \quad \alpha_{2}=0, \quad \alpha_{3}=C(1 \quad v)+4 \mu_{S}, \quad \alpha_{4}=\alpha_{s} C\left(\begin{array}{lll}
1 & v
\end{array}\right), \\
& \beta_{1}=D v+h^{2} \lambda_{S} / 2, \quad \beta_{2}=0, \quad \beta_{3}=D(1 \quad v)+h^{2} \mu_{S}, \quad \beta_{4}=\alpha_{t} D(1 \quad v), \\
& C^{*}=C+4 \mu_{S}+2 \lambda_{S}, \quad D^{*}=D+h^{2} \mu^{S}+h^{2} \lambda^{S} / 2 .
\end{aligned}
$$

Here $C^{*}$ and $D^{*}$ are the effective in-plane and bending stiffness of the plate with surface stresses. It is clear that $C^{*}>C$ and $D^{*}>D$, i.e. the plate with surface stresses is stiffer. The elastic moduli $\alpha_{4}$ and $\beta_{4}$ do not depend on $\lambda_{S}$ and $\mu_{S}$. Hence, the 2D constitutive equation of the plate with surface stresses is given by (5) with the elastic constants defined by (13). For the values of $\lambda_{S}$ and $\mu_{S}$ presented in Duan et al. (2008) for an aluminium, the calculations (Altenbach et al., 2009) show that the influence of the surface stresses is significant if $h \leqslant 20 \mathrm{~nm}$.

\section{Discussion and conclusions}

The discussions on the application of the continuum mechanics and the mechanics of structures in the nanoscale are very extensive, see Duan et al. (2008) among others. Let us note that even for nanostructures as nanoshells, nanofilms, and nanoplates the continuum approach gives a satisfying coincidence with atomistic simulations, if one takes into account the appropriate constitutive equations, see e.g. (Kudin et al., 2001; Miller \& Shenoy, 2000; Peng et al., 2008). The most popular in nanomechanics are the Kirchhoff, Love, Reissner, Mindlin, and von Kàrmàn theories of plate and shells (see Eremeyev et al., 2009; Huang, 2008; Kudin et al., 2001; Miller \& Shenoy, 2000; Lu et al., 2006). Within the framework of these theories the derivation of the plate or shell equations is based on the approximations of the displacement vector through the thickness coordinate $\zeta$. On the other hand, for the nanosized structures such approximations are under consideration. Another problem of the continuum approach is the determination of the Young's modulus and the Poisson's ratio used in (13) for the nanosized structural elements together with the surface elastic constants. The direct approach to formulation of the constitutive equations of nanoshells seems more perspective because within this approach one should determine 6 constants in (5) only.

In this paper we present the analysis for the nonlinear elastic shells using the approach suggested in Libai and Simmonds (1998), Chróścielewski et al. (2004), where no kinematic assumptions are made (such as the Kirchhoff-Love hypotheses). We derive the representation (10) for the stress and couple stress resultant tensors. The theory is deduced in the more simple case of the infinitesimal strains. The effect of the surface stresses may be more significant for multilayered plates and shells and for plates and shells with fractal-like surface.

\section{Acknowledgment}

The second author was supported by the DFG with the Grant No. AL 341/33-1 and by the RFBR with the Grant No. 09-0100459 .

\section{References}

Altenbach, J., Altenbach, H., \& Eremeyev, V. A. (2010). On generalized Cosserat type theories of plates and shells. A short review and bibliography. Archive of Applied Mechanics, 80, 7392.

Altenbach, H., Eremeyev, V. A., \& Morozov, N. F. (2009). Linear theory of shells taking into account surface stresses. Doklady Physics54, 531535.

Altenbach, H., \& Zhilin, P. A. (1988). A general theory of elastic simple shells (in Russian). Uspekhi Mekhaniki, 11, 107148.

Chen, C. Q., Shi, Y., Zhang, Y. S., Zhu, J., \& Yan, Y. J. (2006). Size dependence of Young's modulus in ZnO nanowires. Physical Review Letters, 96, 075505075514.

Chróścielewski, J., Makowski, J., \& Pietraszkiewicz, W. (2004). Statics and dynamics of multifolded shells. Nonlinear therory and finite element method. IPPT PAN, Warsaw. 
Cosserat, E., Cosserat, F. (1909). Théorie des corps déformables. Herman et Fils, Paris.

Cuenot, S., Frétigny, C., Demoustier Champagne, S., \& Nysten, B. (2004). Surface tension effect on the mechanical properties of nanomaterials measured by atomic force microscopy. Physical Review, B69, 165410165415.

Dahmen, K., Lehwald, S., \& Ibach, H. (2000). Bending of crystalline plates under the influence of surface stress a finite element analysis. Surface Science, 446, 161173.

Duan, H. L., Wang, J., \& Karihaloo, B. L. (2008). Theory of elasticity at the nanoscale. Advances in Applied Mechanics, 42, 168.

Eremeyev, V. A. (2005). Nonlinear micropolar shells: Theory and applications. In W. Pietraszkiewicz \& C. Szymczak (Eds.), Shell structures: Theory and applications (pp. 11 18). London: Taylor \& Francis.

Eremeyev, V. A., Altenbach, H., \& Morozov, N. F. (2009). The influence of surface tension on the effective stiffness of nanosize plates. Doklady Physics54, 98100.

Eremeyev, V. A., \& Pietraszkiewicz, W. (2006). Local symmetry group in the general theory of elastic shells. Journal of Elasticity, 85, 125152.

Eremeyev, V. A., \& Zubov, L. M. (2008). Mechanics of elastic shells (in Russian). Moscow: Nauka.

Eringen, A. C. (1966). Linear theory of micropolar elasticity. Journal of Mathematics and Mechanics, 15, 909923.

Eringen, A. C. (1967). Theory of micropolar plates. ZAMP, 18, 1230.

Eringen, A. C. (1999). Microcontinuum field theory. I. Foundations and solids. New York: Springer.

Finn, R. (1986). Equilibrium capillary surfaces. New York: Springer.

Gibbs, J. W. (1928). On the equilibrium of heterogeneous substances. In: The collected works of J. Willard Gibbs, Longmans (pp. 55353$)$. New York: Green \& Co.

Grigolyuk, E. I., \& Selezov, I. T. (1973). Nonclassical theories of vibration of beams, plates and shells (in Russiian). In: Itogi nauki i tekhniki, volume 5 of Mekhanika tverdogo deformiruemogo tela. VINITI, Moskva.

Grigolyuk, E. I., \& Kogan, A. F. (1972). Present state of the theory of multilayered shells (in Russiian). Prikladnaya Matematika I Mekhanika, 8, 317.

Guo, J. G., \& Zhao, Y. P. (2005). The size dependent elastic properties of nanofilms with surface effects. Journal of Applied Physics, 98, 074306 (11pp.).

Guo, J. G., \& Zhao, Y. P. (2007). The size dependent bending elastic properties of nanobeams with surface effects. Nanotechnology, 18, 295701. 6pp.

Gurtin, M. E., \& Murdoch, A. I. (1975a). A continuum theory of elastic material surfaces. Archive for Rational Mechanics and Analysis, 57, 291323.

Gurtin, M. E., \& Murdoch, A. I. (1975b). Addenda to our paper A continuum theory of elastic material surfaces. Archive for Rational Mechanics and Analysis, 59, 389390 .

He, L. H., Lim, C. W., \& Wu, B. S. (2004). A continuum model for size dependent deformation of elastic films of nano scale thickness. International Journal of Solids and Structures, 41, 847857.

Huang, D. W. (2008). Size dependent response of ultra thin films with surface effects. International Journal of Solids and Structures, $45,568579$.

Jing, G. Y., Duan, H. L., Sun, X. M., Zhang, Z. S., Xu, J., Li, Y. D., et al (2006). Surface effects on elastic properties of silver nanowires: Contact atomic force microscopy. Physical Review, B73, 235409235416.

Kafadar, C. B., \& Eringen, A. C. (1971). Micropolar media I. The classical theory. International Journal of Engineering Science, 9, 271305.

Kafadar, C. B., \& Eringen, A. C. (1976). Polar field theories. In A. C. Eringen (Ed.). Continuum physics (Vol. IV, pp. 1 75). New York: Academic Press.

Kudin, K. N., Scuseria, G. E., \& Yakobson, B. I. (2001). C 2 F, BN and C nanoshell elasticity from ab initio computations. Physical Review, B64, 235406.

Landau, L., \& Lifshitz, E. (1987). Fluid mechanics. Course of theoretical physics (2nd ed.) (Vol. 6). Oxford: Pergamon Press.

Laplace, P. S. (1805). Sur l'action capillaire. supplément à la théorie de l'action capillaire. In: Traité de mécanique céleste. Livre X, Gauthier Villars et fils, Paris. (Vol. 4, Suppl. 1, pp. 771 777).

Laplace, P. S. (1806). À la théorie de l'action capillaire. supplément à la théorie de l'action capillaire. In: Traité de mécanique céleste. Livre X, Gauthier Villars et fils, Paris. (Vol. 4, Suppl. 2, pp. 909 945).

Lebedev, L. P., Cloud, M. J., \& Eremeyev, V. A. (2010). Tensor analysis with applications in mechanics. New Jersey: World Scientific.

Libai, A., \& Simmonds, J. G. (1998). The nonlinear theory of elastic shells (2nd ed.). Cambridge: Cambridge University Press.

Lu, P., He, L. H., Lee, H. P., \& Lu, C. (2006). Thin plate theory including surface effects. International Journal of Solids and Structures, $43,46314647$.

Lu, C. F., Lim, C. W., \& Chen, W. Q. (2009). Size dependent elastic behavior of FGM ultra thin films based on generalized refined theory. International Journal of Solids and Structures, 46, 11761185.

Lurie, A. I. (2005). Theory of elasticity. Foundations of engineering mechanics. Berlin: Springer.

Miller, R. E., \& Shenoy, V. B. (2000). Size dependent elastic properties of nanosized structural elements. Nanotechnology, $11,139147$.

Mindlin, R. (1951). Influence of rotatory inertia and shear on flexural motions of isotropic elastic plates. Transactions of the ASME. Journal of Applied Mechanics, 18, 3138.

Müller, P., \& Saúl, A. (2004). Elastic effects on surface physics. Surface Science Reports, 54, 157258.

Naghdi, P. (1972). The theory of plates and shells. In S. Flügge (Ed.). Handbuch der Physik (Vol. VIa/2, pp. 425 640). Springer.

Orowan, E. (1970). Surface energy and surface tension in solids and fluids. Philosophical Transactions of the Royal Society of London Series A, 316, 473491.

Peng, J., Wu, J., Hwang, K. C., Song, J., \& Huang, Y. (2008). Can a single wall carbon nanotube be modeled as a thin shell? Journal of the Mechanics and Physics of Solids, 56, 22132224.

Pietraszkiewicz, W. (1979a). Consistent second approximation to the elastic strain energy of a shell. ZAMM, $59,206208$.

Pietraszkiewicz, W. (1979b). Finite rotations and langrangian description in the non linear theory of shells, Warszawa Poznań: Polish Sci. Publ.

Pietraszkiewicz, W., \& Eremeyev, V. A. (2009). On natural strain measures of the non linear micropolar continuum. International Journal of Solids and Structures, 46, 774787.

Podstrigach, Y. S., \& Povstenko, Y. Z. (1985). Introduction to mechanics of surface phenomena in deformable solids (in Russian). Kiev: Naukova Dumka.

Reissner, E. (1944). On the theory of bending of elastic plates. Journal of Mathematical Physics, 23, 184194.

Reissner, E. (1945). The effect of transverse shear deformation on the bending of elastic plates. Transactions of the ASME Journal of Applied Mechanics, 12, A69 A77.

Rubin, M. B. (2000). Cosserat theories: Shells. Rods and points. Dordrecht: Kluwer.

Rusanov, A. I. (2005a). Thermodynamics of solid surfaces. Surface Science Reports, 23, 173247.

Rusanov, A. I. (2005b). Surface thermodynamics revisited. Surface Science Reports, 58, 111239.

Shi, Y. J., Guo, W. L., \& Ru, C. Q. (2008). Relevance of Timoshenko beam model to microtubules of low shear modulus. Physica E Low Dimensional Systems \& Nanostructures, 41, 213219.

Steigmann, D. J., \& Ogden, R. W. (1999). Elastic surface substrate interactions. Philosophical Transactions of the Royal Society of London Series A Mathematical Physical and Engineering Sciences, 455, 437474.

Truesdell, C., \& Noll, W. (1965). The nonlinear field theories of mechanics. In S. Flügge (Ed.). Handbuch der Physik (Vol. III/3, pp. 1 602). Berlin: Springer.

Wang, J., Huang, Q. A., \& Yu, H. (2008). Young's modulus of silicon nanoplates at finite temperature. Applied Surface Science, $255,24492455$.

Wang, Z. Q., \& Zhao, Y. P. (2009). Self instabilityand bending behaviors of nano plates. Acta Mechanica Solida Sinica, 22, 630643.

Wang, Z. Q., Zhao, Y. P., \& Huang, Z. P. (2010). The effects of surface tension on the elastic properties of nano structures. International Journal of Engineering Science, 48, 140150.

Wunderlich, W. (1973). Vergleich verschiedener Approximationen der Theorie dünner Schalen (mit numerischen Beispielen), Technical Report 73 1, Techn. Wiss. Mitt. des Institut für Konstruktiven Ingenieurbau, Ruhr Universität Bochum.

Young, T. (1805). An essay on the cohesion of fluids. Philosophical Transactions of the Royal Society of London, 95, 6587. 
Yun, G., \& Park, H. S. (2009). Surface stress effects on the bending properties of fcc metal nanowires. Physical Review, B79, 195421.

Zheng, X.-P., Cao, Y.-P., Li, B., Feng, X.-Q., \& Wang, G.-F. (2010). Surface effects in various bending-based test methods for measuring the elastic property of nanowires. Nanotechnology, 21.

Zhu, H. X. (2010). Size-dependent elastic properties of micro- and nano-honeycombs. Journal of the Mechanics and Physics of Solids, 58, 696-709.

Zhu, H. X., Wang, J. X., \& Karihaloo, B. (2009). Effects of surface and initial stresses on the bending stiffness of trilayer plates and nanofilms. Journal of the Mechanics and Physics of Solids, 4, 589-604. 